GTC-99/12-1

gr-qc/9912032

\title{
Mass of Colored Black Holes
}

\author{
Alejandro Corichi* and Daniel Sudarsky ${ }^{\dagger}$ \\ Instituto de Ciencias Nucleares \\ Universidad Nacional Autónoma de México \\ A. Postal 70-543, México D.F. 04510, México.
}

\begin{abstract}
New results pertaining to colored static black hole solutions to the EinsteinYang-Mills equations are obtained. The isolated horizons framework is used to define the concept of Hamiltonian Horizon Mass of the black hole. An unexpected relation between the ADM and Horizon masses of the black hole solution and the ADM mass of the corresponding Bartnik-McKinnon soliton is found. These results can be generalized to other non-linear theories and they suggest a general testing bed for the instability of the corresponding hairy black holes.
\end{abstract}

Pacs: 04.70.-s, 04.70.Bw

*E-mail: corichi@nuclecu.unam.mx

†E-mail: sudarsky@nuclecu.unam.mx 
The laws of $\mathrm{BH}$ mechanics [1] and an essentially complete set of uniqueness theorems [2.3] have led to a rather complete understanding of the properties of stationary solutions in Einstein-Maxwell-Scalar Field theories [3]. However, with the appearance of new, hairy, black holes in other theories with non-linear matter couplings, such as non-abelian gauge theories, several new issues arose. In particular, the physical significance of the discrete families of colored black holes in, say, the Einstein-Yang-Mills (EYM) system still remains somewhat obscure.

One issue that has been considered in that context is the relation that might exist between the existence of regular static, solitonic solutions and 'hairy' black hole solutions. (This issue has been considered for example in [4] from heuristic and dimensional arguments.)

The purpose of this letter is to shed new light on these issues and to establish unexpected relations between the two classes of solutions that coexist in one theory. We will specifically address the case of static spherically symmetric solutions (SSS) to the EYM equations, but it will be apparent that the methodology can be extended straightforwardly to other cases. The basic input that we use to arrive at these new results comes form the recently formulated "Isolated Horizon" (IH) framework [5, [5]. In particular, two main issues are studied. First, by making a crucial use of the Hamiltonian formulation for Isolated black holes we define the Hamiltonian Horizon Mass (HHM) of SSS black holes in EYM theory. We then use this expression to make the main observation of this letter, namely, to show that this quasilocal definition together with some basic properties of Hamiltonian Mechanics lead us to a formula relating HHM and ADM mass of the colored BH solutions with the ADM mass of the Solitons of the theory. We conclude that the positivity of the 'total energy' spectrum of the colored black holes is related to their instability.

These results are quite surprising, because the IH formalism was developed to extend the notion of black holes to situations where radiation is present - and goes out to infinityand one might have not expected to obtain new results already in the static sector of the theory.

In this note we focus our attention to the Einstein-Yang-Mills system defined by the action,

$$
S_{\mathrm{EYM}}(\mathbf{A})=-\frac{1}{16 \pi} \int_{\mathcal{M}} \sqrt{-g}\left[R+\mathbf{F}_{a b}^{i} \mathbf{F}_{i}^{a b}\right] \mathrm{d}^{4} x,
$$

where the abstract indices $a, b, \ldots$ denote space-time objects and the indices $i, j, \ldots$ are internal indices in the Lie algebra of the gauge group $G$. In this letter we restrict out attention to $G=S U(2)$. The field strength $\mathbf{F}$ is given by $\mathbf{F}_{a b}^{i}=2 \nabla_{[a} \mathbf{A}_{b]}^{i}+\epsilon_{j k}^{i} \mathbf{A}_{a}^{j} \mathbf{A}_{b}^{k}$, that is, the curvature of the Lie algebra valued one form $\mathbf{A}_{a}^{i}$. We note that this theory has a characteristic scale given by a combination of Newton's constant and the coupling constant of the gauge theory. In the present work that scale has been absorbed in the dimension of the coordinates. The equations of motion that follow from $S_{\mathrm{EYM}}$, together with the Bianchi identity for the YM sector, are:

$$
\begin{aligned}
& D_{a} \mathbf{F}^{i a b}=0, \quad D_{[c} \mathbf{F}_{a b]}=0, \\
& R_{a b}=2\left(\mathbf{F}_{a c}^{i} \mathbf{F}_{i b}{ }^{c}-\frac{1}{4} g_{a b} \mathbf{F}^{2}\right),
\end{aligned}
$$


where $\mathbf{F}^{2}=\mathbf{F}_{a b}^{i} \mathbf{F}_{i}^{a b}$, and $D_{a}$ is the generalized covariant derivative defined by $\mathbf{A}$. The dual field tensor is given by ${ }^{*} \mathbf{F}_{a b}=\frac{1}{2} \epsilon_{a b}{ }^{c d} \mathbf{F}_{c d}$, where $\epsilon_{a b c d}$ is the volume form associated with the metric. We can define gauge invariant quantities, for any two-sphere $S$ as follows,

$$
Q_{S}:=\frac{1}{4 \pi} \oint_{S}\left|{ }^{*} \mathbf{F}\right|, \quad P_{S}:=\frac{1}{4 \pi} \oint_{S}|\mathbf{F}|,
$$

where by the two form $|\mathbf{F}|$ we mean the following: take $\epsilon^{a b}$, the area two form associated with the 2-sphere $S$ and define $f^{i}=\mathbf{F}_{a b}^{i} \epsilon^{a b}$. Then $|\mathbf{F}|_{a b}=\sqrt{\sum\left(f^{i}\right)^{2}} \epsilon_{a b}$.

For the results of this letter, the details of the IH analysis in EYM are not necessary. Thus, we only refer to those results of the Hamiltonian formulation that we use for our discussion (details can be found in [7]). In the Einstein-Yang-Mills case the total Hamiltonian consists of a bulk term and two surface terms, one at infinity and the other at the isolated horizon. As usual, the bulk term is a linear combination of constraints and the surface term at infinity yields the ADM energy. In a rest-frame adapted to the horizon it is then natural to identify the surface term at the horizon $\Delta$ as the Horizon Mass, $M_{\Delta}$.

We Consider a foliation of the given space-time region $\mathcal{M}$ by a 1-parameter family of (partial) Cauchy surfaces $M_{t}$, each of which extends from the isolated horizon $\Delta$ to spatial infinity $i^{o}$. Then, the Hamiltonian $H_{t}$ generating evolution along the properly normalized vector field $t^{a}$ that approaches $l^{a}$, the null generator of the horizon $\Delta$, is given by:

$$
\begin{aligned}
H_{t} & =\int_{M} \text { constraints }-M_{\mathrm{ADM}} \\
& +\oint_{S_{\Delta}}\left(\frac{\mu^{-1}}{4 \pi} \Psi_{2}\right)^{2} \epsilon+|(\mathbf{A} \cdot l)| Q_{\Delta}+V,
\end{aligned}
$$

with $\mu$ a normalization factor for the vector field $l^{a}$ generating the horizon chosen as in [6], $|\mathbf{A} \cdot l|$ the norm of $\mathbf{A}_{a}^{i} l^{a}$ and $Q_{\Delta}$ the charge as defined by (田) evaluated at the horizon $\Delta$. As already mentioned, we identify the surface term at $S_{\Delta}$ as the HHM $M_{\Delta}$ of the isolated horizon. The quantity $V$ is constant over the region of phase space being considered in (5), and arises only in the Hamiltonian framework. Using some identities that follow from the IH boundary conditions [6], one can define the surface gravity in terms of the Newman-Penrose component $\Psi_{2}$ and $\mu$. If we now fix the value of $\Phi:=|\mathbf{A} \cdot \ell|$ on $\Delta$ to coincide with the value it takes in the (Abelian) family of static solutions, we can cast $M_{\Delta}$ in a more familiar form:

$$
M_{\Delta}=\frac{1}{4 \pi} \kappa a_{\Delta}+\Phi Q_{\Delta}+V,
$$

where $a_{\Delta}$ is the horizon area. Thus, we obtain a Smarr formula for the mass of the black hole. The quantity $V$ now becomes dependent on the intrinsic -absolutely conserved- parameters of the black hole horizon such as $Q_{\Delta}, P_{\Delta}$ and $a_{\Delta}$, since we now consider the full Isolated Horizon phase space (with all posible values of $Q_{\Delta}, P_{\Delta}, a_{\Delta}$ ). Its functional dependence is not arbitrary but is restricted by the mass variation formula coming from the IH formalism,

$$
\delta M_{\Delta}=\frac{1}{8 \pi} \kappa \delta a_{\Delta}+\Phi \delta Q_{\Delta} .
$$

This equation is a necessary condition for the existence of a consistent Hamiltonian framework, and is sufficient to determine $V$. 
Even when Equation (6) resembles the Smarr formula for static space-times, the meaning of various symbols in the equation is somewhat different. Since an isolated horizon need not be a Killing horizon, in general $M_{\Delta}$ does not equal the ADM mass, nor do $\kappa$ or $\Phi$ refer to a Killing field. Given that the constraints are satisfied in any solution, the bulk term in (5) vanishes as well. Hence, in this case, $H_{t}=M_{\Delta}-E^{\mathrm{ADM}}$, the 'total energy' in the spacetime, i.e., the energy available to be radiated, as is clear from the fact that, in a dynamical process, $\delta H_{t}=\delta E_{\infty}^{\mathrm{Rad}}[6]$. Finally, as emphasized in [6], the matter contribution to the mass formula (6) is subtle: while it does not include the energy in radiation outside the horizon, it does include the energy in the 'Coulombic part' of the field associated with the black hole hair. (Recall that the future limit of the Bondi energy has this property.) This is all the information that we need from the IH formalism. In what follows we restrict our attention to the family of Static Spherically Symmetric (SSS) solutions, as embedded in the IH phase space.

A standard parameterization for the metric and gauge potential is given by,

$$
\begin{aligned}
\mathrm{d} s^{2} & =-N^{2} e^{-2 \delta} \mathrm{d} t^{2}+N^{-2} \mathrm{~d} r^{2}+r^{2} \mathrm{~d} \Omega^{2}, \\
\mathbf{A} & =a \tau_{3} \mathrm{~d} t+\left(w \tau_{1}\right) \mathrm{d} \theta+\left(\cot \theta \tau_{3}+w \tau_{2}\right) \sin \theta \mathrm{d} \phi .
\end{aligned}
$$

with $N^{2}=(1-2 m / r)$ and $\delta, m, a$ and $w$ functions of only $r$. The (constant) matrices $\tau_{1}, \tau_{2}$ and $\tau_{3}$ are the standard basis for $s u(2)$.

Roughly speaking, there are two classes of solutions. The first one are Abelian solutions embedded in $\mathrm{SU}(2)$, with electric charge $e$ and magnetic charge $g$. These are precisely the Reissner-Nordstrom solutions given by $m(r)=M-\left(e^{2}+g^{2}\right) / 2 r$ and $\delta=0$.

The second, and more interesting sector, corresponds to non-Abelian solutions to the EYM equation of motion, which are known to exist only for the 'magnetic' sector of the theory. Here we find the regular solitonic solutions [8] and the so called colored black hole solutions [9] [1]. The curvature takes the standard form [10],

$$
\begin{aligned}
\mathbf{F}= & w^{\prime} \tau_{1} \mathrm{~d} r \wedge \mathrm{d} \theta+w^{\prime} \tau_{2} \sin \theta \mathrm{d} r \wedge \mathrm{d} \phi \\
& -\left(1-w^{2}\right) \tau_{3} \sin \theta \mathrm{d} \theta \wedge \mathrm{d} \phi .
\end{aligned}
$$

In this case, the equations are known to have a discrete number of solutions, for each value of the horizon area, labeled by an integer $n$ that represents the number of nodes of the function $w(r)$. The lowest mode, $n=0$, represents the Schwarzschild solution. Therefore, the solution can be completely parametrized by two numbers $\left(M_{\mathrm{ADM}}, n\right)$, the ADM mass and the integer $n$.

From an historical perspective, these were the first examples of 'hairy black holes' . They are 'hairy' because the electric and magnetic charges defined at infinity are both zero, so the only parameter at infinity is the ADM mass. If the no-hair conjecture were valid for the EYM system, the specification of $M_{\mathrm{ADM}}$ would suffice to characterize the solution completely. However, this is not the case, since for a given value of the ADM mass, there exist a countable number of different solutions, labeled by $n$.

Let us now evaluate the HHM for the special case of colored black holes. These solutions are purely magnetic, so $Q_{\Delta}=0$. The formula for the mass (6) now takes the form,

$$
M_{\Delta}=\frac{1}{4 \pi} \kappa a_{\Delta}+V\left(r_{\Delta}, P_{\Delta}\right),
$$


where

$$
\kappa=\frac{e^{-\delta\left(r_{\Delta}\right)}}{2 r_{\Delta}}\left[1-\frac{P_{\Delta}^{2}}{r_{\Delta}^{2}}\right] \quad \text { and } \quad a_{\Delta}=4 \pi r_{\Delta}^{2} .
$$

(recall that $\delta(\infty)=0$.) The magnetic charge $P_{\Delta}$, for the colored black holes (8) is given by: $P_{\Delta}^{2}=\left(1-w_{\Delta}^{2}\right)^{2}$. We expect this formula to reduce to the Smarr formula for the $n=0$ solution, since in that case $\delta \equiv 0$ and $w= \pm 1$, so $m_{\Delta}=r_{\Delta} / 2$. Thus, for $n=0$, we expect $V \equiv 0$.

The mass variation formula (7) coming from the isolated horizons framework, when restricted to the purely magnetic sector of the SSS space takes the form,

$$
\delta M_{\Delta}=\frac{1}{8 \pi} \kappa \delta a_{\Delta}
$$

which is the first law for the HHM.

Now, in order to have a consistent Hamiltonian formulation, one should be able to integrate (12) to find a function $M_{\Delta}$. A detailed analysis shows that we have a consistent Hamiltonian formulation if and only if (11) and (12) are compatible. This in turn implies that $a_{\Delta}$ and $P_{\Delta}$ are not free to vary independently; their variations are constrained to lie in one dimensional subspaces of the $\left(a_{\Delta}, P_{\Delta}\right)$ plane [12]. We can then view the magnetic charge $P_{\Delta}$ as a function of $r_{\Delta}$. This fact is, of course, verified in the explicit numerical solutions reported in the literature. Next, we can arrive at the condition that the function $V$ should satisfy. If we write $\kappa\left(r_{\Delta}\right)=\beta\left(r_{\Delta}\right) /\left(2 r_{\Delta}\right)$ it takes the form,

$$
V^{\prime}=-\frac{r_{\Delta}}{2} \beta^{\prime}
$$

with 'prime' denoting differentiation with respect to $r_{\Delta}$. Furthermore, by requiring that $M_{\Delta} \mapsto 0$ as $r_{\Delta} \mapsto 0$, -coming from physical considerations- we arrive at the following relation,

$$
M_{\Delta}=\frac{1}{2} \int_{0}^{r_{\Delta}} \beta\left(\tilde{r}_{\Delta}\right) \mathrm{d} \tilde{r}_{\Delta}
$$

where the integration is performed over the space of parameters of the black hole, labeled by the horizon radius $r_{\Delta}$, and not over space-time. This is the first observation of this letter. Let us note that for the $n=0$ solution, where $\beta$ is known in closed form $(\beta=1)$, we arrive at $M_{\Delta}^{(n=0)}=r_{\Delta} / 2=\kappa a_{\Delta} /(4 \pi)$, as expected.

Several remarks are in order. First, we must emphasize that the determination of $V$, and thus of $M_{\Delta}$ relied on considerations involving only variations of quantities associated with the horizon $\Delta$. Second, the HHM defined by (6), when restricted to SSS configurations, does not agree with the usual definitions of mass that one finds in the literature (see for instance [13] and [14]). It should be stressed that (14) comes from a consistent Hamiltonian formulation, and is not a definition as occurs in other treatments.

There is a general argument from symplectic geometry that states that, within each connected component of the SSS space embedded in the space of isolated horizons, the value of the Hamiltonian $H_{t}$ remains constant [6]. Let us review this argument since it is essential for our discussion. The Hamilton equations of motion can be written as $\delta H=\Omega\left(\delta, X_{H}\right)$, 
where $\Omega$ is the symplectic form, $\delta$ is an arbitrary variation and $X_{H}$ is the Hamiltonian vector field. A static solution is one at which the Hamiltonian vector field either vanishes or generates pure gauge evolution. In either case, the symplectic structure evaluated on $X_{H}$ and any arbitrary vector field $\delta$ vanishes. Therefore, for this point of the phase space, $\delta H=0$ for any direction $\delta$. In particular $\delta H=0$ for variations relating two static solutions. Now, in the case of Einstein-Maxwell theory, the no-hair theorems ensure that all static solutions are given by the RN family. That is, the space of static solutions is in that case, connected. Furthermore, since there is no energy scale in the theory, the only possible value for $H_{t}$ is zero [6].

What is the situation in Einstein-Yang-Mills theory? First, there is the Abelian family of solutions, that represent a connected component, parametrized by $M, Q, P$. For these solutions, the basic reasoning of [6] applies, with subtle modifications pertaining to the magnetic solutions [7]. Second, as we mentioned above, the EYM system possesses an energy scale, so in principle, non-zero values of $H$ are allowed. Each connected component of the space of SSS colored black holes is one-dimensional (parametrized by $r_{\Delta}$ ), and solutions corresponding to distinct values of $n$ belong to disconnected components. That is, the space SSS has a countable number of connected components. As we shall now show, for $n \geq 1$ the value of the Hamiltonian turns out to be different from zero: $H_{t}^{n} \neq 0$.

Recall that the general argument described above tells us that the (on shell) value of the Hamiltonian is constant for each family labeled by $n$. This in particular implies that its value is independent of the radius $r_{\Delta}$ of the horizon. Thus one is allowed to take the limit

$$
H^{(n)}=\lim _{r_{\Delta} \mapsto 0}\left[M_{\mathrm{ADM}}^{(n)}\left(r_{\Delta}\right)-M_{\Delta}^{(n)}\left(r_{\Delta}\right)\right] .
$$

Now, it is known that the colored black holes converge point-wise to the Bartnik-McKinnon soliton solutions [8] and that the ADM mass satisfies $M_{\mathrm{ADM}}^{(n)} \mapsto M_{\mathrm{BK}}^{(n)}$ when $r_{\Delta} \mapsto 0$. Furthermore, the mass of the black hole goes to zero in this limit, so we can conclude that

$$
H^{(n)}=M_{\mathrm{BK}}^{(n)}
$$

that is, the total value of the Hamiltonian equals the mass of the $n$th Bartnik-McKinnon soliton solution!

We now collect our results and arrive at the unexpected relation,

$$
M_{\mathrm{ADM}}^{(n)}\left(r_{\Delta}\right)=M_{\mathrm{BK}}^{(n)}+M_{\Delta}^{(n)}\left(r_{\Delta}\right) .
$$

Thus, we are in the position of writing an explicit formula for the ADM mass of the $n$ colored black hole as function of $r_{\Delta}$,

$$
M_{\mathrm{ADM}}^{(n)}\left(r_{\Delta}\right)=M_{\mathrm{BK}}^{(n)}+\frac{1}{2} \int_{0}^{r_{\Delta}} \beta^{(n)}\left(\tilde{r}_{\Delta}\right) \mathrm{d} \tilde{r}_{\Delta} .
$$

This formula has been numerically tested for the first colored black holes, finding complete agreement [7]. This is the second observation of this note. It is important to stress that, a priori, one would not expect to get the value of quantities defined at infinity, like the difference of ADM masses in terms of purely local quantities at $\Delta$. 
We can now try to understand the physical meaning of the relation (18). Two facts are known about these solutions: first, we know that for fixed $a_{\Delta}$ these solutions represent saddle points of the ADM mass function $M$ [15], and thus, as one can expect, for all values of $n$ these solutions are unstable under small perturbations [16]. Let us now note that for the reported solutions in the literature (see, for instance, [8]), the BK Mass is a monotonic function of $n$, starting at $M_{\mathrm{BK}}^{1} \approx 0.828$ and approaching 1 as $n$ grows (in standard normalized units). The fact that the mass of the soliton, and therefore the total energy of the colored black holes is positive, confirms our expectation, coming from energetic considerations, that in general $M^{\mathrm{ADM}} \geq M_{\Delta}$. Furthermore, as the difference between the HHM and ADM mass can be seen as the energy that is available for radiation to fall both into the black hole and to infinity, one can understand the nonzero value of the Hamiltonian as an indication that there is a potentiality for instability of the solution. That is, a necessary condition for the solution to be unstable is for the value of Hamiltonian on the solution in question to be positive.

Let us end with two remarks. First, note that the first law for the HHM (đ) is consistent with the results given by different formalisms at infinity, where it has been shown that the ADM mass varies as [15,114],

$$
\delta M_{\mathrm{ADM}}=\frac{1}{8 \pi} \kappa \delta a_{\Delta},
$$

but since we know that $\delta H=\delta\left(M_{\mathrm{ADM}}-M_{\Delta}\right)=0$, we have complete agreement with (12). Finally, let us note that it should be possible to apply the type of analysis presented here to other theories where nontrivial static solutions have been found. In particular, Einstein Yang Mills Higgs, Einstein Yang Mills Dilaton, and Einstein Skyrme Theories, are examples in which there are both solitonic and Black Hole solutions.

We would like to thank A. Ashtekar, C. Beetle, S. Fairhurst and U. Nucamendi for discussions, R. Wald for correspondence, and the Center for Gravitational Physics and Geometry for its hospitality. This work was in part supported by DGAPA-UNAM grant No IN121298, by a NSF-CONACyT colaborative grant, by NSF grants INT9722514, PHY95-14240 and by the Eberly research funds of Penn State. 


\section{REFERENCES}

[1] J.W. Bardeen, B. Carter and S.W. Hawking, The four laws of black hole mechanics Commun. Math. Phys. 31, 161 (1973).

[2] Chruściel P., No Hair Theorems Folklore, Conjectures, Results, Contemporary Mathematics 170, 23 (1994).

[3] M. Heusler, Black Hole Uniqueness Theorems (Cambridge University Press, Cambridge, 1996)

[4] P. Bizon, Gravitating solitons and hairy black holes, Acta Phys. Polon. B 25, 877 (1994).

[5] A. Ashtekar, A. Corichi and K. Krasnov, Isolated Horizons: The Classical Phase Space, Adv. Theor. Math. Phys. 3, 418 (1999). (Preprint gr-qc/9905089.)

[6] A. Ashtekar, C. Beetle and S. Fairhurst, Mechanics of isolated horizons, Class. Quantum Grav. 17, 253 (2000).

[7] A. Corichi, U. Nucamendi and D. Sudarsky, Einstein-Yang-Mills Isolated Horizons: Phase Space, Mechanics, Hair and Conjectures, (Preprint gr-qc/0002078).

[8] R. Bartnik and J. McKinnon, Particlelike Solutions to the Einstein-Yang-Mills Equations, Phys. Rev. Lett. 61, 141 (1988).

[9] M. S. Volkov and D. V. Gal'tsov, Non-Abelian Einstein-Yang-Mills black holes, JETP Lett. 50, 346 (1989).

[10] P. Bizon, Colored Black Holes, Phys. Rev. Lett. 64, 2844 (1990).

[11] H.P. Kunzle and A.K.M. Masood-ul-Alam, Spherically symmetric static SU(2) EinsteinYang-Mills fields, J. Math. Phys. 31, 928 (1990).

[12] C. Beetle and A. Corichi, Preprint-CGPG (2000).

[13] M. S. Volkov and D. V. Gal'tsov D V Gravitating Non-Abelian solitons and black holes with Yang-Mills fields, Physics Reports 319, 1-83 (1999).

[14] M. Heusler and N. Straumann, The first law of black hole physics for a class of non-linear matter models, Class. Quantum Grav. 10, 1299 (1993).

[15] D. Sudarsky and R. Wald, Extrema of mass, stationarity, and staticity, and solutions to the Einstein-Yang-Mills equations, Phys. Rev. D 46, 1453 (1992).

[16] N. Straumann and Z. H. Zhou, Instability of a colored black hole solution, Phys. Lett. B243, 33 (1990). 